\title{
Pacific
}

Journal of

Mathematics

\section{THE NEUMANN PROBLEM ON LIPSCHITZ DOMAINS IN HARDY SPACES OF ORDER LESS THAN ONE}

\author{
RUSSELL M. BROWN
}




\title{
THE NEUMANN PROBLEM ON LIPSCHITZ DOMAINS IN HARDY SPACES OF ORDER LESS THAN ONE
}

\author{
Russell M. BROWN
}

\begin{abstract}
Recently, B.E.J. Dahlberg and C.E. Kenig considered the Neumann problem, $\Delta u=0$ in $D, \partial u / \partial \nu=f$ on $\partial D$, for Laplace's equation in a Lipschitz domain $D$. One of their main results considers this problem when the data lies in the atomic Hardy space $H^{1}(\partial D)$ and they show that the solution has gradient in $L^{1}(\partial D)$. The aim of this paper is to establish an extension of their theorem for data in the Hardy space $H^{p}(\partial D), 1-\epsilon<p<1$, where $0<\epsilon<1 / n$ is a positive constant which depends only on $m$, the maximum of the Lipschitz constants of the functions which define the boundary of the domain. We also extend G. Verchota's and Dahlberg and Kenig's theorem on the potential representation of solutions of the Neumann problem to the range $1-\epsilon<p<1$. This has the interesting consequence that the double-layer potential is invertible on Hölder spaces $C^{\alpha}(\partial D)$ for $\alpha$ close to zero.
\end{abstract}

The techniques of this paper are a modification of those of Dahlberg and Kenig [6]. In Lemma 2.10 of [6], Varopoulos's extension lemma and $H^{1}(\partial D)$ $V M O(\partial D)$ duality are used to show that a harmonic function with nontangential maximal function in $L^{1}(\partial D)$ has normal derivative in $H^{1}(\partial D)$. This argument fails when $p<1$, since we cannot realize $H^{p}(\partial D)$ as a dual space. To substitute for the use of their Lemma 2.10, we observe that solutions of the Dirichlet problem with $H_{1}^{p}(\partial D)$-data have normal derivative in $H^{p}(\partial D)$. This follows from Dahlberg and Kenig's construction. Then, we need to prove a uniqueness result in order to know that the functions produced by the single-layer potential are identical to the functions constructed in their existence theorem. We remark that we are also able to give a direct proof that $M(\nabla u) \in L^{p}(\partial D)$ implies $\partial u / \partial \nu \in H^{p}(\partial D)$ when $(n-1) / n<p \leq 1$. This is done using atomic decomposition techniques of M. Wilson [16]. We remark that, after seeing a preliminary version of this paper, Wei Cao and E. Fabes [1] established similar results on the invertibility of the potential operators using an extension of the techniques in [2].

\section{Existence.}

We let $D \subset \mathbf{R}^{n}$ denote a connected Lipschitz domain. Thus for every $Q \in$ $\partial D$, there is an $r>0$, a coordinate system on $\mathbf{R}^{n}$ and a Lipschitz function 
$\phi: \mathbf{R}^{n-1} \rightarrow \mathbf{R}$ with $\phi(0)=0$ and such that

$$
Z(Q, 100 r) \cap \partial D=\left\{\left(X^{\prime} X_{n}\right): X_{n}=\phi\left(X^{\prime}\right)\right\} \cap Z(Q, 100 r)
$$

and

$$
Z(Q, 100 r) \cap D=\left\{\left(X^{\prime}, X_{n}\right): X_{n}>\phi(X)\right\} \cap Z(Q, 100 r)
$$

where

$$
Z(Q, s)=\left\{\left(X^{\prime}, X_{n}\right):\left|X^{\prime}-Q^{\prime}\right|<s,\left|X_{n}-Q_{n}\right|<(1+2 m) s\right\} .
$$

We call $Z(Q, s)$ a coordinate cylinder for $\partial D$. We note that since $\partial D$ is compact, we may assume that $\partial D$ is covered by a finite collection of coordinate cylinders whose radii $r$ are bounded below by $r_{0}$.

Our results will only be proven for starshaped Lipschitz domains in $\mathbf{R}^{n}$, $n \geq 3$. This means that, after a translation, $0 \in \Omega$ and if $X \in \Omega$, then $r X \in \Omega$ for $0 \leq r<1$. These assumptions are inherited from the work of Dahlberg and Kenig. It is easier to prove Theorems A and B quoted below for these special domains. It is not difficult to extend these results to more general domains, but we do not discuss this extension here.

We let $\Delta\left(Q_{0}, r\right)=\left\{P \in \partial D:\left|P-Q_{0}\right|<r\right\}$ and assume that $r$ is less than $\operatorname{diam}(\partial D)$. We let $d=n-1$ denote the dimension of $\partial D$. We say that $a$ is an atom for $H^{p}(\partial D)$ if for some $Q_{0}$ and $r$ we have

i) $\operatorname{supp} a \subset \Delta\left(Q_{0}, r\right)$

ii) $\int_{\Delta\left(Q_{0}, r\right)} a(Q) d Q=0$

iii) $\|a\|_{L^{2}\left(\Delta\left(Q_{0}, r\right)\right)} \leq c r^{-d(1 / p-1 / 2)}$.

When $1 \geq p>\frac{d}{d+1}$, the space $H^{p}(\partial D)$ is defined as the collection

$$
\left\{f: f=\sum \lambda_{j} a_{j} \text { with } \sum \lambda_{j}^{p}<\infty\right\}
$$

for some sequence of atoms $a_{j}$. The quasi-norm for $H^{p}(\partial D)$ given by

$$
\|f\|_{H^{p}(\partial D)}^{p}=\inf \left\{\sum \lambda_{j}^{p}: f=\sum \lambda_{j} a_{j}\right\} .
$$

We note that the infinite sums appearing here do not exist as functions. Rather one must view elements of $H^{p}(\partial D)$ as linear functionals on spaces of nice functions. In fact, the dual of $H^{p}(\partial D), d /(d+1)<p<1$, is the space of Hölder continuous function of exponent $\alpha(p)=d(1-p) / p$. Thus, the pairing between an element of $H^{p}(\partial D)$ and $C^{\alpha(p)}(\partial D)$ is defined. We will abuse 
notation by writing this pairing as an integral $\int_{\partial D} f u d Q$ for $f \in H^{p}(\partial D)$, and $u \in C^{\alpha(p)}(\partial D)$. We recall that $H^{p}(\partial D)$ is not a Banach space since the triangle inequality fails. However, we may define a metric on $H^{p}(\partial D)$ by $\|f-g\|_{H^{p}(\partial D)}^{p}$.

In studying the exterior Neumann problem, it will be useful to introduce the space $\tilde{H}^{p}(\partial D)$. This is defined in the same manner as $H^{p}(\partial D)$, but we include the atom $\chi_{\partial D}$. We let $C^{\alpha}(\partial D), 0<\alpha<1$, denote the collection of equivalence classes of Hölder continuous functions which differ by a constant. The norm is given by

$$
\|f\|_{C^{\alpha}(\partial D)}=\sup _{Q \neq P} \frac{|\bar{f}(P)-\bar{f}(Q)|}{|P-Q|^{\alpha}}
$$

where the $\bar{f}$ is any representative of $f$. Finally, we define $\tilde{C}^{\alpha}(\partial D)$ as the space of functions for which the norm $\|f\|_{\tilde{C}^{\alpha}(\partial D)}=\|f\|_{L^{\infty}(\partial D)}+\|f\|_{C^{\alpha}(\partial D)}$ is finite. We let $C_{0}^{\alpha}(A)$ denote the set of functions in $C^{\alpha}(A)$ which have a compactly supported representative.

We study the following boundary value problems:

$$
(N P) \quad\left\{\begin{array} { l l } 
{ \Delta u = 0 , } & { \text { in } D } \\
{ \frac { \partial u } { \partial \nu } = f , } & { \text { on } \partial D }
\end{array} \quad \text { (DP) } \quad \left\{\begin{array}{ll}
\Delta u=0, & \text { in } D \\
u=f, & \text { on } \partial D
\end{array}\right.\right.
$$

Since we will consider boundary values in (NP) which are not functions, we need to define the sense in which $\partial u / \partial \nu$ exists at the boundary. Let $f \in H^{p}(\partial D)$. We say that $\partial u / \partial \nu=f$ on $\partial D$ if for each coordinate cylinder $Z$ and compactly supported function $\psi \in C^{\alpha}(\partial D \cap Z), \alpha=d(1 / p-1)$, we have

$$
\lim _{\epsilon \rightarrow 0^{+}} \int_{\partial D \cap Z} \psi(Q) \frac{\partial u_{\epsilon}}{\partial \nu}(Q) d Q=\int_{\partial D} \psi(Q) f(Q) d Q
$$

where $u_{\epsilon}(X)=u\left(X+\epsilon e_{n}\right)$ is defined in a neighborhood of $Z \cap D$. We will also need to define tangential derivatives at the boundary. Let $Z, \phi$ be a coordinate cylinder. If $f$ is smooth in a neighborhood of $Z \cap \partial D$, then we define tangential derivatives by

$$
\frac{\partial f}{\partial T_{i}}\left(X^{\prime}, \phi\left(X^{\prime}\right)\right)=\frac{\partial}{\partial X_{i}} f\left(X^{\prime}, \phi\left(X^{\prime}\right)\right), \quad i=1, \ldots, n-1 .
$$

If $u$ is smooth in $D$, we say that $\nabla_{t a n} u$ exists in the $H^{p}(\partial D)$ sense if for each coordinate cylinder $Z$, there exists $f_{1}, \ldots, f_{n-1} \in H^{p}(\partial D)$ so that for all $\psi \in C_{0}^{\alpha}(\partial D \cap Z)$

$$
\lim _{\epsilon \rightarrow 0^{+}} \int_{\partial D \cap Z} \frac{\partial u}{\partial T_{i}}\left(Q+s e_{n}\right) \psi(Q) d Q=\int_{\partial D} f_{i} \psi, \quad i=1, \ldots, n-1 .
$$


An important part of the argument for inverting the layer potentials is the study of the Dirichlet problem when the data has one derivative in $H^{p}(\partial D)$. We call this space $H_{1}^{p}(\partial D)$ and give a precise definition by defining atoms. We say that $A$ is an atom for $H_{1}^{p}(\partial D)$ if for some $Q_{0} \in \partial D$ and $r>0$, we have

i) $\operatorname{supp} A \subset \Delta\left(Q_{0}, r\right) \cap \partial D$

ii) $\left\|\nabla_{t a n} A\right\|_{L^{2}(\partial D)} \leq r^{-d(1 / p-1 / 2)}$

and then define $H_{1}^{p}(\partial D)$ as the $l^{p}$-span of these atoms. We note that our definition of $H_{1}^{1}(\partial D)$ is slightly different than the one given in [6]. However, it is easy to see that the resulting spaces coincide.

We begin by stating Dahlberg and Kenig's existence results for solutions with atomic data. Their results for $H^{1}(\partial D)$ have a little wiggle room so they also apply to $H^{p}(\partial D)$. To state these results, we will use the nontangential maximum function. For a function $v$ which is continuous on $D$, this is defined by

$$
M(v)(P)=\sup _{X \in \Gamma(P)}|v(X)|
$$

where $\Gamma(P)$ is the nontangential approach region

$$
\Gamma(P)=\left\{Y \in D:|Y-P|<2 \sqrt{1+m^{2}} \delta(Y)\right\}
$$

and $\delta(Y)$ denotes the distance from $Y$ to the boundary of $D$.

Theorem A. Let a be an atom for $H^{p}(\partial D)$ and suppose that a is supported in $\Delta\left(Q_{0}, r\right)$. There exists $\eta>0$ such that if $p>2 /(\eta+2)$, then there is a unique solution of $(N P)$ which satisfies
i) $\int_{\partial D} M(\nabla u)(P)^{2} d P \leq C r^{-d(2 / p-1)}$
ii) $\int_{\partial D} M(\nabla u)(P)^{2}\left|P-Q_{0}\right|^{d(1+\eta)} d P \leq c r^{d(\eta+2-2 / p)}$

iii) We have that $\left.u\right|_{\partial D} \in H^{p}(\partial D)$ and if we normalize by setting $u(0)=0$, then $\|u\|_{H_{1}^{p}(\partial D)} \leq C$.

Similarly, if $a \in \tilde{H}^{p}(\partial D)$, is an atom, then the solution of the exterior Neumann problem in $\mathbf{R}^{n} \backslash \bar{D}$ satisfies i) and ii). If we normalize by requiring $u$ to vanish at infinity, then we obtain the estimate of iii) also.

We also quote the corresponding result for $H_{1}^{p}(\partial D)$-atoms.

Theorem B. Let $A$ be an atom for $H_{1}^{p}(\partial D)$ which is supported in $\Delta\left(Q_{0}, r\right)$. There exists $\eta>0$ such that if $p>2 /(\eta+2)$, then the $L_{1}^{2}$-solution of the Dirichlet problem with data $A$ satisfies

i) $\int_{\partial D} M(\nabla u)(P)^{2} d P \leq C r^{-d(2 / p-1)}$ 
ii) $\int_{\partial D} M(\nabla u)(P)^{2}\left|P-Q_{0}\right|^{d(1+\eta)} d P \leq C r^{d(\eta+2-2 / p)}$

iii) $\partial u / \partial \nu \in H^{p}(\partial D)$ and $\|\partial u / \partial \nu\|_{H^{p}(\partial D)} \leq C$

iv) $u$ has tangential derivatives in the $H^{p}$-sense.

Theorem A and B are established in [6]. The estimates iii) are not explicitly stated in their paper. However, they follow easily from i) and ii) via the idea of a molecule (see [4]). The statements for the exterior problems may be obtained from the interior problems (in a different domain) using the Kelvin transform.

As immediate corollaries of Theorems A and B, we obtain the solvability of the boundary value problems with data in $H_{1}^{p}(\partial D)$ and $H^{p}(\partial D)$. In these theorems and in much of the rest of this article, we will restrict $p$ to the range $1-\delta_{m}<p \leq 1$ where $1 / n>\delta_{m}>0$ is determined by the following three conditions: 1) $\delta_{m}<1-2 /(\eta+2)$ where $\eta$ is as in Theorems A and B. 2) If $p>1-\delta_{m}$, then we must be able to solve the Dirichlet problem with data in the dual space, $C^{\alpha(p)}(\partial D), \alpha(p)=d(1 / p-1)$, and obtain a solution in $C^{\alpha(p)}(\bar{D})$ (see Lemma 2.3). 3) The Neumann Green's function for domains lying above the graph of a function with Lipschitz constant $m$ must lie in $C^{\alpha(p)}$ away from the singularity. See [6] or Theorem 2.8 below for the construction of this Green's function.

Theorem C. Let $1>p>1-\delta_{m}$ and suppose that $f \in H^{p}(\partial D)$. Then the interior Neumann problem with data $f$ has a solution $u$ which satisfies

$$
\|u\|_{H_{1}^{p}(\partial D)}+\|M(\nabla u)\|_{L^{p}(\partial D)} \leq C\|f\|_{H^{p}(\partial D)}
$$

when $u$ is normalized by $u(0)=0$. For the exterior problem, we allow $f$ in $\tilde{H}^{p}(\partial D)$ and we normalize by setting $u(\infty)=0$. This solution satisfies the estimate

$$
\|u\|_{H_{1}^{p}(\partial D)}+\|M(\nabla u)\|_{L^{p}(\partial D)} \leq C\|f\|_{\tilde{H}^{p}(\partial D)} .
$$

Furthermore, the normal and tangential derivatives of $u$ exist in the sense described above.

Theorem D. Let $1-\delta_{m}<p<1$ and suppose $f \in H_{1}^{p}(\partial D)$. Then the interior Dirichlet problem with data $f$ has a solution in $D$ which satisfies

$$
\|M(\nabla u)\|_{L^{p}(\partial D)}+\left\|\frac{\partial u}{\partial \nu}\right\|_{H^{p}(\partial D)} \leq C\|f\|_{H^{p}(\partial D)} .
$$

For the exterior problem, we have

$$
\|M(\nabla u)\|_{L^{p}(\partial D)}+\left\|\frac{\partial u}{\partial \nu}\right\|_{\tilde{H}^{p}(\partial D)} \leq C\|f\|_{H^{p}(\partial D)}
$$


In each case, the normal and tangential derivatives exist in the sense described above.

We close this section with a theorem whose proof is due to M. Wilson. We observe that if $u$ is harmonic in a Lipschitz domain $D, M(\nabla u)$ lies in $L^{p}(\partial D)$, $p>(n-1) / n$, then we may define $\partial u / \partial \nu$ as a linear functional on Lipschitz functions on the boundary. In fact, if $\psi$ is supported in a coordinate cylinder $Z$, then

$$
\lim _{\epsilon \rightarrow 0^{+}} \int_{\partial D \cap Z} \psi(Q) \frac{\partial u}{\partial \nu}\left(Q+\epsilon e_{n}\right) d Q=\lim _{\epsilon \rightarrow 0^{+}} \int_{D} \nabla \psi(Y) \nabla u\left(Y+\epsilon e_{n}\right) d Y .
$$

Using Lemma 2.1 below, one can see that

$$
\int_{\partial D \cap Z}\left|\nabla u\left(Q+r e_{n}\right)\right| d Q \leq r^{-d(1-p) / p}\|M(\nabla u)\|_{L^{p}(\partial D)} .
$$

Hence, for $p>(n-1) / n$ the integral on the right of (1.1) converges as $\epsilon \rightarrow 0^{+}$ (see also the proof of Theorem 2.9).

Theorem 1.2. Let $(n-1) / n<p \leq 1$ and suppose that $u$ is harmonic with $M(\nabla u) \in L^{p}(\partial D)$, then we may find an atomic decomposition of $\partial u / \partial \nu$ into $H^{p}(\partial D)$ atoms. In particular, $\partial u / \partial \nu$ is in $H^{p}(\partial D)$. For the exterior domain, we obtain the normal derivative is in $\tilde{H}^{p}(\partial D)$.

This may be proven using the techniques of M. Wilson from [16]. His argument works without alteration in domains lying above the graph of a Lipschitz function. We leave the details of general domains to the reader. We note that this theorem provides a different proof of the estimates for the normal derivative in Theorem $\mathrm{D}$. It would be interesting to see if the estimates of the boundary values of $u$ in Theorem $\mathrm{C}$ can be obtained this way.

\section{Uniqueness.}

In this section, we show that the solutions described in section one are unique. This also depends on the ideas developed in [6]. However, there are some technical difficulties in dealing with the case $p<1$. Our main new tool is Lemma 2.2 which allows us to estimate the $L^{1}$-norm of $M(u)$ in terms of the $L^{p}$-norm of $M(\nabla u), p=(n-1) / n$. This is a version of the Hardy-Littlewood theorem on fractional integration. In Lemma 2.2 below, we prove a sharp version of this result. We will show that for harmonic $u$, we can always control $\|M(u)\|_{p d /(d-p)}$ by $\|M(\nabla u)\|_{p}$, when $p<n-1=d$. 
In our first lemma, we let $f_{E} f$ denote the average $|E|^{-1} \int_{E} f$. Similar results are known for classical Hardy spaces. In [9], Krantz proves a fractional integration result for atomic Hardy spaces. Earlier, Stein and Weiss observe that the theorem on fractional integration holds for the Hardy spaces which they define in [13]. In one dimension, the result dates back to Hardy and Littlewood.

Lemma 2.1. Let $w$ be harmonic in $D$ and let $Z$ be a coordinate cylinder and let $\alpha$ be a multi-index with nonnegative entries, then

$$
\left|\frac{\partial^{\alpha} w}{\partial X^{\alpha}}(X)\right| \leq C \delta(X)^{-d / p-|\alpha|}\|M(w)\|_{L^{p}(\partial D)} .
$$

Proof. Using interior estimates for harmonic functions, we have

$$
\begin{aligned}
\frac{\partial^{\alpha} w}{\partial X^{\alpha}}(X) & \leq \frac{C_{p}}{\delta(X)^{|\alpha|}}\left(f_{B(X, \delta(X) / 2)}|\nabla w(Y)|^{p} d Y\right)^{1 / p} \\
& \leq C \delta(X)^{-|\alpha|}\left(f_{\Delta(\hat{X}, C \delta(X))} M(\nabla w)(Q)^{p} d Q\right)^{1 / p} \\
& \leq C_{p, m, \alpha} \delta(X)^{-d / p-|\alpha|}\|M(\nabla w)\|_{p}
\end{aligned}
$$

where $\hat{X}$ denotes a point on $\partial D$ satisfying $\delta(X)=|X-\hat{X}|$.

Lemma 2.2. Let $D$ be a connected Lipschitz domain and suppose that $u$ is harmonic in $D$. Let $X^{*}$ be a fixed point in $D$ and suppose that $u\left(X^{*}\right)=0$. For $p<d$ and $p^{*}=d p /(d-p)$ we have

$$
\|M(u)\|_{L^{p^{*}}(\partial D)} \leq C\|M(\nabla u)\|_{L^{p}(\partial D)}
$$

where the constant $C$ depends on the distance of $X^{*}$ to the boundary, $p$ and the Lipschitz character of $\partial D$.

Proof. We prove the corresponding result for a domain

$$
D=\left\{\left(X^{\prime}, X_{n}\right): X_{n}>\phi\left(X^{\prime}\right)\right\}
$$

which lies above the graph of a Lipschitz function $\phi$. From Lemma 2.1, we have $|\nabla u(X)| \leq C \delta(X)^{-d / p}$. It follows that $\lim _{X_{n} \rightarrow \infty} u\left(X^{\prime}, X_{n}\right)$ exists and is independent of $X^{\prime}$. Thus we may add a constant to $u$ and obtain that $u$ vanishes at infinity. Also, after replacing $u$ by $u_{\epsilon}(X)=u\left(X+\epsilon e_{n}\right)$, we may assume that $M(u) \in L^{p^{*}}(\partial D)$. 
We will need the area integral which is defined by

$$
A(u)(Q)^{2}=\int_{\Gamma^{\prime}(Q)}|\nabla u(Y)|^{2}|Y-Q|^{2-n} d Y
$$

where $\Gamma^{\prime}(Q) \supset \Gamma(Q)$ is a strictly larger cone defined by

$$
\left\{X \in D: \delta(X)<4 \sqrt{1+m^{2}}|X-Q|\right\} .
$$

To estimate $M(u)$, we use the fundamental theorem of calculus and Hölder's inequality to obtain that for each $\eta>0$,

$$
\begin{aligned}
\left|u\left(X^{\prime}, X_{n}\right)\right| \leq & \int_{X_{n}}^{\infty}\left|\frac{\partial u}{\partial X_{n}}\left(X^{\prime}, s\right)\right| d s \\
\leq & \left(\int_{X_{n}}^{\infty}\left(s-\phi\left(X^{\prime}\right)\right)\left|\nabla u\left(X^{\prime}, s\right)\right|^{2} d s\right)^{\frac{1}{2} 2 \eta} \\
& \times\left(\int_{X_{n}}^{\infty}\left(s-\phi\left(X^{\prime}\right)\right)^{-\eta /(1-\eta)}\left|\nabla u\left(X^{\prime}, s\right)\right|^{\frac{1-2 \eta}{1-\eta}} d s\right)^{\frac{1-\eta}{1-2 \eta}(1-2 \eta)} \\
\equiv & B_{1}(X)^{2 \eta} B_{2}(X)^{1-2 \eta}
\end{aligned}
$$

Since $B_{1}(X)$ is essentially the $g$-function, we have $B_{1}(X) \leq C A(u)(Q)$ for $X \in \Gamma(Q)$.

To study the function $B_{2}(X)$, we let $B_{X^{\prime}, s}$ denote the ball $B\left(\left(X^{\prime}, s\right), c_{m}[s-\right.$ $\left.\left.\phi\left(X^{\prime}\right)\right]\right)$ where $c_{m}$ is chosen so that $B_{X^{\prime}, s}$ lies in $D$ and we let $\Delta_{X^{\prime}, s}=$ $\Delta\left(Q, C\left[s-\phi\left(X^{\prime}\right)\right]\right)$. If we choose $C$ sufficiently large, then we have

$$
\begin{aligned}
B_{2}(X)^{\frac{1-2 \eta}{1-\eta}} & \leq C_{\eta} \int_{X_{n}}^{\infty}\left(s-\phi\left(X^{\prime}\right)\right)^{-\eta /(1-\eta)} f_{B_{X^{\prime}, s}}|\nabla u(Y)|^{\frac{1-2 \eta}{1-\eta}} d Y d s \\
& \leq C_{\eta, m} \int_{X_{n}}^{\infty}\left(s-\phi\left(X^{\prime}\right)\right)^{-\eta /(1-\eta)} f_{\Delta_{X^{\prime}, s}} M(\nabla u)(P)^{\frac{1-2 \eta}{1-\eta}} d P d s .
\end{aligned}
$$

Changing the order of integration in this last integral, we obtain

$$
\begin{aligned}
B_{2}(X)^{\frac{1-2 \eta}{1-\eta}} & \leq C \int_{\partial D} M(\nabla u)(P)^{\frac{1-2 \eta}{1-\eta}}|P-Q|^{\frac{1-2 \eta}{1-\eta}-d} d P \\
& \equiv F(Q)^{\frac{1-2 \eta}{1-\eta}}, \quad X \in \Gamma(Q) .
\end{aligned}
$$

By the Hardy-Littlewood theorem on fractional integration [12, p. 119], we have

$$
\|F\|_{L^{p^{*}}(\partial D)} \leq C\|M(\nabla u)\|_{L^{p}(\partial D)}
$$

when $p<d, \frac{1}{2}>\eta>0$ and, if $p \leq 1, \eta>(1-p) /(2-p)$. Combining our estimates for $B_{1}$ and $B_{2}$, we have

$$
\|M(u)\|_{L^{p^{*}}(\partial D)} \leq C\|A(u)\|_{L^{p^{*}}(\partial D)}^{2 \eta}\|M(\nabla u)\|_{L^{p}(\partial D)}^{1-2 \eta} .
$$


Now, we may use Dahlberg's estimate [5] for the area integral, $\|A(u)\|_{L^{p}(\partial D)}$ $\leq C\|M(u)\|_{L^{p}(\partial D)}$, and our a priori assumption that $M(u)$ is in $L^{p^{*}}(\partial D)$ to obtain the estimate of the theorem.

Our final preliminary result studies the action of the generalized Riesz transforms on $C^{\alpha}(\partial D)$ when $D$ is a Lipschitz domain. We restrict our attention to domains lying above the graph of a Lipschitz function and introduce the notation $D_{\phi}=\left\{\left(X^{\prime}, X_{n}\right): X_{n}>\phi\left(X^{\prime}\right)\right\}$ with $\|\nabla \phi\|_{\infty}=m$.

Lemma 2.3. Let $\phi: \mathbf{R}^{n-1} \rightarrow \mathbf{R}$ be Lipschitz. There exists $\alpha_{0}$ depending only on $m$ such that for $f \in C^{\alpha}\left(\partial D_{\phi}\right), 0<\alpha<\alpha_{0}$, we may find a harmonic gradient $\left(w^{1}, \ldots, w^{n}\right)$ satisfying $w^{n}(Q)=f(Q)$,

$$
\begin{aligned}
\frac{\partial w^{i}}{\partial X_{j}} & =\frac{\partial w^{j}}{\partial X_{i}} \quad i, j=1, \ldots, n \\
\sum_{i=1}^{n} \frac{\partial w^{i}}{\partial X_{i}} & =0 \\
\Delta w^{i} & =0, \quad i=1, \ldots, n .
\end{aligned}
$$

Furthermore, each of these functions is Hölder continuous in $\bar{D}_{\phi}$ and satisfies

$$
\left\|w^{i}\right\|_{C^{\alpha}\left(\bar{D}_{\phi}\right)} \leq C\|f\|_{C^{\alpha}\left(\partial D_{\phi}\right)}, \quad i=1, \ldots, n .
$$

This is fairly standard, thus our proof will be brief.

Proof. We let $w^{n}$ be the solution of the Dirichlet problem in $D_{\phi}$ with data $f$. We have $\left\|w^{n}\right\|_{C^{\alpha}\left(\bar{D}_{\phi}\right)} \leq C\|f\|_{C^{\alpha}\left(\partial D_{\phi}\right)}$. Next, we apply interior estimates to the harmonic function $w^{n}(\cdot)-w^{n}(X)$ on the ball $B(X, \delta(X) / 2)$ to obtain that

$$
\left|\frac{\partial^{\beta} w^{n}}{\partial X^{\beta}}(X)\right| \leq C \delta(X)^{\alpha-|\beta|}\left\|w^{n}\right\|_{C^{\alpha}\left(\bar{D}_{\phi}\right)}, \quad|\beta| \geq 1 .
$$

The converse also holds for any function which is in $C_{\text {loc }}^{1}\left(D_{\phi}\right)$ :

$$
\|u\|_{C^{\alpha}\left(\bar{D}_{\phi}\right)} \leq C_{\alpha, m} \sup _{X \in D_{\phi}} \delta(X)^{1-\alpha}|\nabla u(X)| .
$$

We can define the conjugate functions by the formula

$$
\nabla w^{i}(X)=-\int_{X_{n}}^{\infty} \nabla \frac{\partial w^{n}}{\partial X_{i}}\left(X^{\prime}, s\right) d s .
$$

The estimate (2.4) guarantees that this integral is converges and (2.5) implies the functions $w^{2}$ are Hölder continuous. 
Lemma 2.6. If $\left(w^{1}, \ldots, w^{n}\right)$ is a harmonic gradient satisfying $w^{n} \geq 0, w^{n}$ vanishes continuously on $\partial D \backslash \Delta\left(Q_{0}, r\right)$, and $\lim _{X_{n} \rightarrow \infty} w^{i}\left(X^{\prime}, X_{n}\right)=0$ for each $i$ then

$$
\sup \left\{\left|w^{i}(X)\right|: \operatorname{dist}\left(X, \Delta\left(Q_{0}, r\right)\right) \geq r\right\} \leq C_{r, q}\left\|M\left(w^{n}\right)\right\|_{q}
$$

Proof. We first observe that Chebyshev's inequality implies that

$$
\left|w^{n}\left(Q_{0}+r e_{n}\right)\right| \leq C_{r}\left\|M\left(w^{n}\right)\right\|_{L^{q}(\partial D)} .
$$

Using the boundary Harnack principle (see [8, Lemma 5.4], for example), we obtain that for some $\alpha>0$

$$
w^{n}(X) \leq C_{r, \alpha}\left(\frac{\delta(X)}{r}\right)^{\alpha} w^{n}\left(Q_{0}+r e_{n}\right), \quad X \in D \backslash B\left(Q_{0}, 2 r\right) .
$$

Applying the maximum principle in $D_{\phi} \backslash B\left(Q_{0}, 2 r\right)$ yields that $w^{n}$ is bounded there.

To obtain the boundedness of $w^{i}$ we observe that (2.7) implies that

$$
\left|\nabla w^{n}(X)\right| \leq C_{r, \alpha} \delta(X)^{\alpha-1}\left\|M\left(w^{n}\right)\right\|_{q}, X \in D_{\phi} \backslash B\left(Q_{0}, 2 r\right) .
$$

While Lemma 2.1 gives

$$
\left|\nabla w^{n}(X)\right| \leq C_{q, m} \delta(X)^{-1-d / q}\left\|M\left(w^{n}\right)\right\|_{L^{q}(\partial D)} .
$$

Using these estimates and writing $w^{n}$ as an integral of its derivative,

$$
w^{i}(X)=-\int_{X_{n}}^{\infty} \frac{\partial w^{n}}{\partial X_{i}}\left(X^{\prime}, s\right) d s
$$

gives the boundedness of the functions $w^{i}$.

We are now ready to give our uniqueness result for the Neumann problem.

Theorem 2.8 (Uniqueness in NP). If $1-\delta_{m}<p<1$, u satisfies

$$
\left\{\begin{array}{l}
\Delta u=0 \\
M(\nabla u) \in L^{p}(\partial D)
\end{array}\right.
$$

and $\partial u / \partial \nu$ vanishes in the $H^{p}$-sense, then $u$ is a constant.

Proof. We fix a coordinate cylinder $(Z, \phi)$ and let $X^{*}=\left(X^{\prime}, 2 \phi\left(X^{\prime}\right)-X_{n}\right)$ be reflection in the graph of $\phi$. We let $G(X, Y)$ be the Green's function $G(X, Y)+G\left(X, Y^{*}\right)=N(X, Y)$ be the Neumann kernel for $D_{\phi}$ constructed 
in [6, p. 447]. We have that $N(X, Q) \in C^{\alpha}(\partial D)$ for $0<\alpha<\alpha(m)$. This follows since $G(X, Y)$ is the fundamental solution in $\mathbf{R}^{n}$ of an operator whose coefficients are bounded and measurable $[\mathbf{1 0}, \mathbf{1 1}]$.

We let $\psi$ be a cutoff function satisfying $\chi_{1 / 2 Z} \leq \psi \leq \chi_{Z}$. We have

$$
\begin{array}{rl}
\psi(X) u_{\epsilon}(X)=\int_{D} & N(X, Y) u_{\epsilon}(Y) \Delta \psi(y) d Y \\
& +2 \int_{D} N(X, Y) \nabla u_{\epsilon}(Y) \nabla \psi(Y) d Y \\
& +\int_{\partial D} N(X, Q) u_{\epsilon}(Q) \frac{\partial \psi}{\partial \nu}(Q) d Q \\
& +\int_{\partial D} N(X, Q) \frac{\partial u_{\epsilon}(Q)}{\partial \nu} \psi(Q) d Q \\
= & A(X)+B(X)+C(X)+D(X) .
\end{array}
$$

To establish uniqueness, we first show that $u$ is bounded in $\frac{1}{4} Z$. We observe that $N(X, Y) \leq C\left[|X-Y|^{2-n}+\left|X^{*}-Y\right|^{2-n}\right]$ for $X \in D,[\mathbf{1 0}]$. Thus, we have

$$
|C(X)| \leq \int_{Z \cap \partial D}\left|u_{\epsilon}(Q)\right| d Q \operatorname{dist}\left(X,\left(\frac{1}{2} Z\right)^{c}\right)^{2-n} . \quad X \in \frac{1}{4} Z
$$

Next, we observe that Lemma 2.1 implies that

$$
\begin{aligned}
\int_{Z \cap D}\left|\nabla u_{\epsilon}\right| d X & \leq C\left\|M\left(\nabla u_{\epsilon}\right)\right\|_{p}^{1-p} \int_{0}^{2 r_{0}} \int_{\partial D} M\left(\nabla u_{\epsilon}\right)(P) d P r^{-d(1-p) / p} d r \\
& \leq C\|M(\nabla u)\|_{p}, \quad \text { when } p>(d-1) / d .
\end{aligned}
$$

This gives

$$
|B| \leq C\left\|M\left(\nabla u_{\epsilon}\right)\right\|_{p} \cdot \operatorname{dist}\left(X, 1 / 2 Z^{c}\right)^{2-n} .
$$

The term $D$ vanishes as $\epsilon \rightarrow 0^{+}$because the normal derivative vanishes in the $H^{p}$-sense. This follows because $N(X, \cdot)$ is in $C^{\alpha}(\partial D)$. The term $A$ is easy to estimate and we omit the details. The estimates on $A$ through $C$ and the vanishing of $D$ imply that $u$ is bounded.

To see that $u$ is constant, we choose $f$ in the Hardy space $H^{1}(\partial D)$. By Theorem $\mathrm{C}$, there exists a solution $\psi$ to (NP), with data $f$ and $M(\nabla \psi)$ in $L^{1}(\partial D)$. We let $u_{\epsilon}(X)=u((1-\epsilon) X)$ and consider

$$
\int_{\partial D} u_{\epsilon}(Q) \frac{\partial \psi_{\eta}}{\partial \nu}(Q)-\psi_{\eta}(Q) \frac{\partial u_{\epsilon}}{\partial \nu}(Q) d Q=0
$$

We note that

$$
\int \partial u_{\epsilon} / \partial \nu \psi_{\eta} \rightarrow 0
$$


as $\epsilon \rightarrow 0^{+}$since $\psi_{\eta}$ is smooth. Also,

$$
\int u_{\epsilon} \partial \psi_{\eta} / \partial \nu \rightarrow \int u \partial \psi_{\eta} / \partial \nu
$$

since our claim that $u$ is bounded implies that $u$ has nontangential limits a.e, (see [8], for example). Finally,

$$
0=\int_{\partial D} u \frac{\partial \psi_{\eta}}{\partial \nu} \rightarrow \int_{\partial D} u \frac{\partial \psi}{\partial \nu} d Q
$$

as $\eta \rightarrow 0^{+}$because $u$ is bounded and $\left\|\partial_{\nu} \psi_{\eta}-\partial_{\nu} \psi\right\|_{L^{1}(\partial D)}$. Thus we have shown that

$$
\int_{\partial D} u f=0
$$

for every $f \in H^{1}(\partial D)$. Finally, we know that bounded harmonic functions in Lipschitz domains are the Poisson integral of their boundary values. Hence, $u$ is constant.

Theorem 2.10. Suppose that $\Delta u=0, M(\nabla u) \in L^{p}(\partial D)$ and $p$ lies between $1-\delta_{m}$ and 1 . If the tangential derivatives of $u$ vanish in the $H^{p}$-sense and the nontangential limits of $u$ vanish on $\partial D$, then $u=0$ in $D$.

Proof. We will begin by showing that for each nonnegative $f \in C_{0}^{\alpha}(Z \cap \partial D)$, we have

$$
\lim _{\epsilon \rightarrow 0^{+}} \int_{\partial D} \frac{\partial u_{\epsilon}}{\partial \nu}(Q) f(Q) d Q
$$

exists and the limit satisfies

$$
\left|\int_{\partial D} \frac{\partial u}{\partial \nu} f d Q\right| \leq\|M(w)\|_{L^{2}(\partial D)} \cdot\|M(\nabla u)\|_{L^{p}(\partial D)}
$$

where $w$ is the solution of $\Delta w=0, w=f$ on $\partial D$. Since $\|M(w)\|_{2} \leq C\|f\|_{2}$, the second inequality implies that $\partial u / \partial \nu$ is in $L^{2}(\partial D)$.

Towards establishing (2.11) and (2.12), we fix a coordinate cylinder $Z$ and let $f \in C_{0}^{\alpha}(\partial D \cap Z)$ be nonnegative. We choose a smooth cutoff function $\psi$ which satisfies $\chi_{2 Z} \leq \psi \leq \chi_{4 Z}$. For $\eta, \epsilon>0$ we have

$$
\begin{aligned}
& \int_{\partial D} \psi w_{\epsilon} \frac{\partial u_{\eta}}{\partial \nu} d Q \\
& =\int_{\partial D} u_{\eta} \frac{\partial \psi}{\partial \nu} w_{\epsilon}+u_{\eta} \psi \frac{\partial w_{\epsilon}}{\partial \nu} d Q \\
& \quad-\int_{D} u_{\eta} w_{\epsilon} \Delta \psi+2 u_{\eta} \nabla \psi \cdot \nabla w_{\epsilon} d X \\
& \equiv \int_{\partial D} A(Q)+B(Q) d Q-\int_{D} C_{1}(X)+C_{2}(X) d X
\end{aligned}
$$


We observe that $\frac{\partial \psi}{\partial \nu}$ is supported in $(4 Z \backslash 2 Z) \cap \partial D$ hence

$$
A(Q) \leq M(u)(Q)\|M(w)\|_{2}
$$

by Lemma 2.6. To bound the integrand, $C_{2}(X)$, we note that $\nabla \psi$ is supported in $4 Z \backslash 2 Z$, hence we may use the observation of Lemma 2.6 that $|\nabla w(X)| \leq C \delta(X)^{\alpha-1}\|M(w)\|_{2}$ in $4 Z \backslash 2 Z$ to estimate

$$
C_{2}\left(Q+r e_{n}\right) \leq M(u)(Q) r^{\alpha-1}\left\|M\left(w^{n}\right)\right\|_{L^{2}(\partial D)} .
$$

The estimate for $C_{1}(X)$ is also easy.

This leaves the main term $\int_{\partial D} B$ to be understood. We let $w^{n}, w^{n-1}, \ldots, w^{1}$ be the harmonic gradient determined by $f$ (see Lemma 2.3) and write

$$
\begin{aligned}
\int_{\partial D} \psi(Q) u_{\eta}(Q) \frac{\partial w_{\epsilon}^{n}}{\partial \nu}(Q) d Q & \\
= & \int_{\mathbf{R}^{n-1}} \psi\left(X^{\prime}, \phi\left(X^{\prime}\right)\right) u_{\eta}\left(X^{\prime}, \phi\left(X^{\prime}\right)\right)\left(-\frac{\partial w_{\epsilon}^{n}}{\partial X_{n}}\left(X^{\prime}, \phi\left(X^{\prime}\right)\right)\right. \\
& \left.\quad+\sum_{i=1}^{n-1} \phi_{X_{i}}\left(X^{\prime}\right) \frac{\partial w_{\epsilon}^{n}}{\partial X_{i}}\left(X^{\prime}, \phi\left(X^{\prime}\right)\right)\right) d X^{\prime} \\
= & \int_{\mathbf{R}^{n-1}} \psi\left(X^{\prime}, \phi\left(X^{\prime}\right)\right) u_{\eta}\left(X^{\prime}, \phi\left(X^{\prime}\right)\right)\left(\sum_{i=1}^{n-1} \frac{\partial}{\partial X_{i}}\left[w_{\epsilon}^{i}\left(X^{\prime}, \phi\left(X^{\prime}\right)\right)\right]\right) d X^{\prime} \\
= & -\int_{\partial D} w_{\epsilon}^{i}(Q) u_{\eta}(Q) \frac{\partial \psi}{\partial T_{i}}(Q)+\psi(Q) w_{\epsilon}^{i}(Q) \frac{\partial u_{\eta}}{\partial T_{i}}(Q) d Q \\
\equiv & -\int_{\partial D} B_{1}(Q)+B_{2}(Q) d Q .
\end{aligned}
$$

By Lemma 2.6, we have

$$
B_{1}(Q) \leq M(u)(Q)\left\|M\left(w^{n}\right)\right\|_{L^{2}(\partial D)}
$$

Our hypothesis that $\partial u / \partial T_{i}$ vanishes implies that $\int B_{2}$ vanishes as $\epsilon \rightarrow 0^{+}$. Thus, we may let $\epsilon \rightarrow 0^{+}$in each of these expressions and then let $\eta$ go to zero to obtain (2.11) and (2.12). This uses Lemma 2.2 to bound the $L^{1}$-norm of $M(u)$.

Our next step is to show that the nontangential maximal function of $u$ is in $L^{2}(\partial D)$. This and our assumption that $u$ has nontangential limits of 0 a.e. on $\partial D$ are sufficient to imply that $u$ vanishes identically on $\partial D$.

As in Theorem 2.8, we fix a coordinate cylinder $Z$, a cutoff function $\psi$ and let $N(X, Y)$ be the Neumann Green's function for a graph domain $D_{\phi}$ 
for which $D \cap 2 Z=D_{\phi} \cap 2 Z$. We let $u_{\epsilon}(X)=u\left(X+\epsilon e_{n}\right)$ and apply Green's formula to obtain

$$
\begin{aligned}
\psi u_{\epsilon}(X)= & \int_{\partial D} N(X, Q)\left(\psi(Q) \frac{\partial u_{\epsilon}}{\partial \nu}+u_{\epsilon}(Q) \frac{\partial \psi}{\partial \nu}(Q)\right) d Q \\
& +\int_{D} N(X, Y)\left(u_{\epsilon}(Y) \Delta \psi(Y)+\nabla u_{\epsilon}(Y) \cdot \nabla \psi(Y)\right) d Y
\end{aligned}
$$

We have just shown that we may let $\epsilon \rightarrow 0^{+}$in the term involving $\partial u_{\epsilon} / \partial \nu$. Since Lemma 2.2 implies $M\left(u_{\epsilon}\right) \in L^{1}(\partial D)$ and as in the proof of Theorem 2.8 we have $\nabla u_{\epsilon}$ and $u_{\epsilon}$ in $L^{1}(D)$, we may let $\epsilon \rightarrow 0^{+}$in the representation formula for $\psi u_{\epsilon}$ and obtain

$$
\begin{aligned}
\psi u(X)= & \int_{\partial D} N(X, Q) \psi(Q) \frac{\partial u}{\partial \nu} d Q \\
& +\int_{D} N(X, Y)(u(Y) \Delta \psi(Y)+\nabla u(Y) \cdot \nabla \psi(Y)) d Y \\
= & A(X)+B(X)
\end{aligned}
$$

As in Theorem 2.8 , the term $B(X)$ is clearly bounded in $1 / 4 Z$, say. To estimate $B(X)$, we use our observation above that $\partial u / \partial \nu$ is in $L^{2}$ of the boundary. In fact, since $N(X, Y) \leq C|X-Y|^{2-n}$ in $D$, it is easy to see that the nontangential maximal function of $A(X)$ is in $L^{2}(D)$. This establishes our claim about $M(u)$ and hence the Theorem follows.

Remark. The calculation used to estimate the term $B$ in the study of $\partial u / \partial \nu$ was used by G. Verchota in [15].

\section{Layer potentials.}

In this section, we show that the solutions of the Neumann problem constructed in Section 2 may also be represented as single-layer potentials. This representation follows from the estimates of Theorem $C$ and $D$ via an argument of G. Verchota $[\mathbf{1 4}, \mathbf{1 5}]$. Using the potential representation of the solutions of the Neumann problem, we immediately obtain a potential representation for solutions of the Dirichlet problem with data in $C^{\alpha}(\partial D)$ (or $\tilde{C}^{\alpha}(\partial D)$ for the exterior Dirichlet problem).

We begin by defining these potentials and recalling their mapping properties. We let

$$
\Gamma(X)=\frac{1}{(n-2) \omega_{n}|X|^{n-2}}
$$

denote the fundamental solution of Laplace's equation in $\mathbf{R}^{n}, n \geq 3$. Here, $\omega_{n}$ denotes the volume of the unit ball in $\mathbf{R}^{n}$. We define the single-layer 
potential of $f \in H^{p}(\partial D)$, by

$$
\mathcal{S}(f)(X)=\int_{\partial D} \Gamma(X-Q) f(Q) d Q, X \in \mathbf{R}^{n} \backslash \partial D .
$$

Note that we may also define $\mathcal{S}(f)$ on $\partial D$ as an element of $L^{p d /(d-p)}(\partial D)$. This is the familiar Hardy-Littlewood theorem on fractional integration which extends easily to the setting of atomic Hardy spaces. Next, we define the double-layer potential by

$$
\mathcal{D}(f)(X)=\int_{\partial D} \frac{\partial \Gamma}{\partial \nu(Q)}(X-Q) f(Q) d Q .
$$

Notice that if $X \notin \bar{D}$, then

$$
\int_{\partial D} \frac{\partial \Gamma}{\partial \nu(Q)}(X-Q) d Q=\int_{D} \Delta_{Y} \Gamma(X-Y) d Y=0 .
$$

Thus the double-layer potential of an equivalence class $\{f(Q)+r: r \in \mathbf{R}\}$ in $C^{\alpha}(\partial D)$ is well-defined. To discuss the boundary values of the potentials, we introduce the boundary potential operators:

$$
\mathcal{K}^{*}(f)(P)=\text { p.v. } \int_{\partial D} \frac{\partial \Gamma}{\partial \nu(P)}(P-Q) f(Q) d Q
$$

and

$$
\mathcal{K}(f)(P)=\text { p.v. } \int_{\partial D} \frac{\partial \Gamma}{\partial \nu(Q)}(P-Q) f(Q) d Q .
$$

The boundedness of $\mathcal{K}: H^{p}(\partial D) \rightarrow H^{p}(\partial D)$ (and on $\tilde{H}^{p}(\partial D)$ ) is a consequence of the results of Coifman, Meyer and McIntosh [3] on the Cauchy integral on Lipschitz curves (see also $[\mathbf{7}, 14]$ ).

Hence, we let $\nu=\nu^{+}$denote the outer normal to $D$ and $\nu^{-}=-\nu^{+}$denote the outer normal to $D^{*}=R^{n} \backslash \partial D$. We let $\mathcal{S}^{+}(f)$ and $\mathcal{S}^{-}(f)$ denote the restrictions of $\mathcal{S}(f)$ to $D$ and $D^{*}$ respectively. Similarly, we let $\mathcal{D}^{+}(f)$ and $\mathcal{D}^{-}(f)$ denote the restrictions of $\mathcal{D}(f)$ to $D$ and $D^{*}$. We summarize the boundary behavior of these operators in our next two results.

Theorem 3.1. Let $p>\frac{d}{d+1}=\frac{n-1}{n}$. Then we have

$$
\begin{aligned}
& \frac{\partial \mathcal{S}^{+}(f)}{\partial \nu^{+}}=\frac{1}{2} f+\mathcal{K}^{*}(f), \quad f \in H^{p}(\partial D) \\
& \frac{\partial \mathcal{S}^{-}(f)}{\partial \nu^{-}}=\frac{1}{2} f-\mathcal{K}^{*}(f), \quad f \in \tilde{H}^{p}(\partial D)
\end{aligned}
$$




$$
\begin{aligned}
\left\|\left(\frac{1}{2} I+\mathcal{K}^{*}\right)(f)\right\|_{H^{p}(\partial D)}+\left\|M\left(\nabla \mathcal{S}^{+}(f)\right)\right\|_{L^{p}(\partial D)} & \|\mathcal{S}(f)\|_{H_{1}^{p}(\partial D)} \\
& \leq C\|f\|_{H^{p}(\partial D)} \\
\left\|\left(\frac{1}{2} I-\mathcal{K}^{*}\right)(f)\right\|_{\tilde{H}^{p}(\partial D)}+\left\|M\left(\nabla \mathcal{S}^{-}(f)\right)\right\|_{L^{p}(\partial D)}+ & \|\mathcal{S}(f)\|_{H_{1}^{p}(\partial D)} \\
& \leq C\|f\|_{\tilde{H}^{p}(\partial D)} .
\end{aligned}
$$

The normal derivatives and the tangential derivatives exist at the boundary in the sense described in Section 1.

Theorem 3.2. Let $0<\alpha<1$ and suppose that $f \in \tilde{C}^{\alpha}(\partial D)$, then we have that

$$
\begin{aligned}
& \left.\mathcal{D}^{+}(f)\right|_{\partial D}=\frac{1}{2} f+\mathcal{K}(f) \text { a.e. } \\
& \left.\mathcal{D}^{-}(f)\right|_{\partial D}=-\frac{1}{2} f+\mathcal{K}(f) \text { a.e. }
\end{aligned}
$$

and we may redefine $\mathcal{K}(f)$ so that these equalities hold everywhere. Furthermore, we have

$$
\left\|\mathcal{D}^{+}(f)\right\|_{\tilde{C}^{\alpha}(\partial D)} \leq C\|f\|_{\tilde{C}^{\alpha}(\partial D)}
$$

and

$$
\left\|\mathcal{D}^{-}(f)\right\|_{C^{\alpha}(\partial D)} \leq C\|f\|_{C^{\alpha}(\partial D)} .
$$

Finally, we have that $\frac{1}{2} I+\mathcal{K}: \tilde{C}^{\alpha}(\partial D) \rightarrow \tilde{C}^{\alpha}(\partial D)$ is the adjoint of $\frac{1}{2} I+$ $\mathcal{K}^{*}: \tilde{H}^{p}(\partial D) \rightarrow \tilde{H}^{p}(\partial D)$ and that $-\frac{1}{2} I+\mathcal{K}: C^{\alpha}(\partial D) \rightarrow C^{\alpha}(\partial D)$ is the adjoint of $-\frac{1}{2} I+\mathcal{K}^{*}: H^{p}(\partial D) \rightarrow H^{p}(\partial D)$ when $\alpha$ and $p$ are related by $\alpha=d\left(\frac{1}{p}-1\right)$.

The next result uses the ideas of G. Verchota $[14,15]$ to establish our main estimate.

Proposition 3.3. Let $D$ be a starshaped Lipschitz domain, then we have

$$
\|f\|_{H^{p}(\partial D)} \leq C\left\|\left(\frac{1}{2} I+\mathcal{K}^{*}\right)(f)\right\|_{H^{p}(\partial D)}
$$

and

$$
\|f\|_{\tilde{H}^{p}(\partial D)} \leq C\left\|\left(\frac{1}{2} I-\mathcal{K}^{*}\right)(f)\right\|_{\tilde{H}^{p}(\partial D)}
$$


Proof. We consider the first estimate. From Theorem 3.1, and the uniqueness results of Theorems 2.8 and 2.10, we see that the estimates of Theorem $\mathrm{C}$ and D apply to $\mathcal{S}(f)$ in $D$ and $D^{*}$. Thus

$$
\begin{aligned}
\|f\|_{H^{p}(\partial D)} & \leq\left\|\left(\frac{1}{2} I+\mathcal{K}^{*}\right)(f)\right\|_{H^{p}(\partial D)}+\left\|\left(\frac{1}{2} I-\mathcal{K}^{*}\right)(f)\right\|_{H^{p}(\partial D)} \\
& \leq\left\|\left(\frac{1}{2} I+\mathcal{K}^{*}\right)(f)\right\|_{H^{p}(\partial D)}+C\|\mathcal{S}(f)\|_{H_{1}^{p}(\partial D)} \\
& \leq C^{\prime}\left\|\left(\frac{1}{2} I+\mathcal{K}^{*}\right)(f)\right\|_{H^{p}(\partial D)} .
\end{aligned}
$$

The first inequality is the triangle inequality, the second is Theorem $\mathrm{C}$ and the third is Theorem D. The proof of the second estimate of our theorem is similar.

We are now ready to give our representation theorem for solutions of the Dirichlet problem with $C^{\alpha}$ data.

Theorem 3.4. Let $D$ be a starshaped Lipschitz domain and let $1-\epsilon<p<1$. Then the maps

$$
\begin{aligned}
& \frac{1}{2} I+\mathcal{K}^{*}: H^{p}(\partial D) \rightarrow H^{p}(\partial D) \\
& \frac{1}{2} I-\mathcal{K}^{*}: \tilde{H}^{p}(\partial D) \rightarrow \tilde{H}^{p}(\partial D)
\end{aligned}
$$

are invertible.

Proof. The estimate of Proposition 3.3 implies that $\frac{1}{2} I+\mathcal{K}^{*}$ is injective and has closed image. Thus, to establish the invertibility, we only need show that the image of $\frac{1}{2} I+\mathcal{K}^{*}$ is dense in $H^{p}(\partial D)$. But this is easy since it is known $[\mathbf{1 4}, \mathbf{1 5}]$ that $\frac{1}{2} I+\mathcal{K}^{*}$ is invertible on $L_{0}^{2}(\partial D) \equiv L^{2}(\partial D) \cap$ $\left\{f: \int f=0\right\}$.

Corollary 3.5. The maps

$$
+\frac{1}{2} I+\mathcal{K}: C^{\alpha}(\partial D) \rightarrow C^{\alpha}(\partial D)
$$

and

$$
-\frac{1}{2} I+\mathcal{K}: \tilde{C}^{\alpha}(\partial D) \rightarrow \tilde{C}^{\alpha}(\partial D)
$$

are invertible for $0<\alpha<d \epsilon /(1-\epsilon)$ where $\epsilon$ is as in Theorem 3.4.

Proof. This follows immediately from Theorem 3.4 and the duality relations stated in Theorem 3.2. 


\section{Acknowledgments.}

I thank E.B. Fabes for gently encouraging me to finish this note and I thank C.E. Kenig for suggesting that Wilson's work [16] would be helpful for obtaining the atomic decomposition of Theorem 1.2.

\section{References}

[1] Wei Cao and E.B. Fabes, Invertibility of an operator on $H_{a t}^{p}$ spaces and Neumann problem on Lipschitz domains, Preprint, 1991.

[2] Wei Cao and Yoram Sagher, Stability and interpolation in families of Banach spaces, preprint, 1990.

[3] R.R. Coifman, A. McIntosh, and Y.Meyer, L'intégrale de Cauchy définit un opérateur borné sur $L^{2}$ pour les courbes lipschitziennes, Ann. of Math., 116 (1982), 361-387.

[4] R.R. Coifman and G. Weiss, Extensions of Hardy spaces and their use in analysis, Bull. Amer. Math. Soc., 83 (1976), 569-645.

[5] B.E.J. Dahlberg, Weighted norm inequalities for the Lusin area integral and nontangential maximal functions for functions harmonic in a Lipschitz domain, Studia Math., 67 (1980), 297-314.

[6] B.E.J. Dahlberg and C.E. Kenig, Hardy spaces and the Neumann problem in $L^{p}$ for Laplace's equation in Lipschitz domains, Ann. of Math., 125 (1987), 437-466.

[7] E.B. Fabes, M. Jodeit, Jr., and N.M. Riviére, Potential techniques for boundary value problems on $C^{1}$-domains, Acta Math., 141 (1978), 165-186.

[8] D.S. Jerison and C.E. Kenig, Boundary value problems in Lipschitz domains, Studies in partial differential equations (Washington, D.C.) (Walter Littman, ed.), MAA Studies in Mathematics, vol. 23, Math. Assoc. Amer., Washington, D.C., 1982, pp. $1-68$.

[9] S.G. Krantz, Fractional integration in Hardy spaces, Studia Math., 73 (1982), 8794.

[10] W. Littman, G. Stampacchia, and H. Weinberger, Regular points for elliptic equations with discontinuous coefficients, Ann. della Sc. N. Sup. Pisa, 17 (1963), 45-79.

[11] J. Moser, On Harnack's theorem for elliptic differential operators, Comm. Pure Appl. Math., 14 (1967), 577-591.

[12] E.M. Stein, Singular integrals and differentiability properties of functions, Princeton University Press, Princeton NJ, 1970.

[13] E.M. Stein and G. Weiss, On the theory of harmonic functions in several variables, I, Acta. Math., 103 (1960), 25-62.

[14] G.C. Verchota, Layer potentials and boundary value problems for laplace's equation on Lipschitz domains, Ph.D. thesis, University of Minnesota, 1982.

[15] - Layer potentials and regularity for the Dirichlet problem for Laplace's equation on Lipschitz domains, J. Funct. Anal., 59 (1984), 572-611.

[16] J. Michael Wilson, A simple proof of the atomic decomposition for $H^{p}\left(R^{n}\right), 0<$ $p \leq 1$, Studia Math., 74 (1982), 25-33. 
Received June 24, 1993. Supported in part by the NSF and the Commonwealth of Kentucky through the Kentucky EPSCoR program.

UNIVERSITY OF KENTUCKY

LEXINGTON, KY 40506-0027 



\title{
PACIFIC JOURNAL OF MATHEMATICS
}

Founded by E. F. Beckenbach (1906-1982) and F. Wolf (1904-1989)

\section{EDITORS}

\author{
Sun-Yung Alice Chang (Managing Editor) \\ University of California \\ Los Angeles, CA 90095-1555 \\ pacific@math.ucla.edu
}

\section{F. Michael Christ}

University of California

Los Angeles, CA 90095-1555

christ@math.ucla.edu

Thomas Enright

University of California

San Diego, La Jolla, CA 92093

tenright@ucsd.edu

Nicholas Ercolani

University of Arizona

Tucson, AZ 85721

ercolani@math.arizona.edu
Robert Finn

Stanford University

Stanford, CA 94305

finn@gauss.stanford.edu

Vaughan F. R. Jones

University of California

Berkeley, CA 94720

vfr@math.berkeley.edu

Steven Kerckhoff

Stanford University

Stanford, CA 94305

spk@gauss.stanford.edu
Martin Scharlemann

University of California

Santa Barbara, CA 93106

mgscharl@math.ucsb.edu

Gang Tian

Courant Institute

New York University

New York, NY 10012-1100

tiang@taotao.cims.nyu.edu

V. S. Varadarajan

University of California

Los Angeles, CA 90095-1555

vsv@math.ucla.edu

\section{SUPPORTING INSTITUTIONS}

\section{CALIFORNIA INSTITUTE OF TECHNOLOGY \\ NEW MEXICO STATE UNIVERSITY \\ OREGON STATE UNIVERSITY \\ STANFORD UNIVERSITY \\ UNIVERSITY OF ARIZONA \\ UNIVERSITY OF BRITISH COLUMBIA \\ UNIVERSITY OF CALIFORNIA UNIVERSITY OF HAWAII}

\author{
UNIVERSITY OF MONTANA \\ UNIVERSITY OF NEVADA, RENO \\ UNIVERSITY OF OREGON \\ UNIVERSITY OF SOUTHERN CALIFORNIA \\ UNIVERSITY OF UTAH \\ UNIVERSITY OF WASHINGTON \\ WASHINGTON STATE UNIVERSITY
}

The supporting Institutions listed above contribute to the cost of publication of this Journal, but they are not owners or publishers and have no responsibility for its contents or policies.

Manuscripts must be prepared in accordance with the instructions provided on the inside back cover.

The Pacific Journal of Mathematics (ISSN 0030-8730) is published monthly except for July and August. Regular subscription rate: $\$ 215.00$ a year (10 issues). Special rate: $\$ 108.00$ a year to individual members of supporting institutions.

Subscriptions, orders for back issues published within the last three years, and changes of subscribers address should be sent to Pacific Journal of Mathematics, P.O. Box 4163, Berkeley, CA 94704-0163, U.S.A. Prior back issues are obtainable from Kraus Periodicals Co., Route 100, Millwood, NY 10546.

The Pacific Journal of Mathematics at the University of California, c/o Department of Mathematics, 981 Evans Hall, Berkeley, CA 94720 (ISSN 0030-8730) is published monthly except for July and August. Second-class postage paid at Berkeley, CA 94704, and additional mailing offices. POSTMASTER: send address changes to Pacific Journal of Mathematics, P.O. Box 6143, Berkeley, CA 94704-0163.

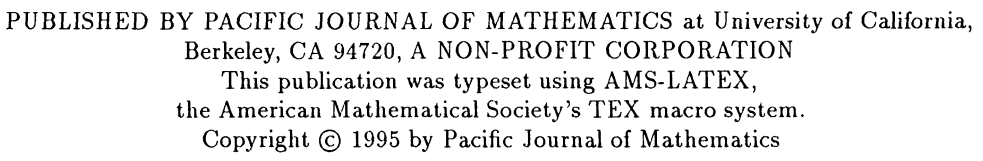




\section{PACIFIC JOURNAL OF MATHEMATICS}

\section{Volume $171 \quad$ No. $2 \quad$ December 1995}

On $H^{p}$-solutions of the Bezout equation

ERIC AMAR, JOAQUIM BRUNA FLORIS and ARTUR NiCOLAU

Amenable correspondences and approximation properties for von Neumann algebras

309

Claire ANANTHARAman-DElaroche

On moduli of instanton bundles on $\mathbb{P}^{2 n+1}$

VinCENZO ANCONA and Giorgio MaRia OtTAVIANI

Minimal surfaces with catenoid ends

JORGEN BERGLUND and WAYNE ROSSMAN

Permutation model for semi-circular systems and quantum random walks

PHILIPPE BIANE

The Neumann problem on Lipschitz domains in Hardy spaces of order less than one

RUSSELL M. BROWN

Matching theorems for twisted orbital integrals

409

REBECCA A. HERB

Uniform algebras generated by holomorphic and pluriharmonic functions on strictly

pseudoconvex domains

ALEXANDER IzZO

Quantum Weyl algebras and deformations of $U(g)$

NAIHUAN JING and JAMES ZHANG

Calcul du nombre de classes des corps de nombres

STÉPHANE LOUBOUTIN

On geometric properties of harmonic Lip ${ }_{1}$-capacity

PertTi MatTila and P. V. PARAmonov

Reproducing kernels and composition series for spaces of vector-valued holomorphic functions

Bent ØRsted and Genkai Zhang

Iterated loop modules and a filtration for vertex representation of toroidal Lie algebras

S. ESWARA RAO

The intrinsic mountain pass

MARTIN SCHECHTER

A Frobenius problem on the knot space

RON G. WANG

On complete metrics of nonnegative curvature on 2-plane bundles

DAVID YANG

Correction to: "Free Banach-Lie algebras, couniversal Banach-Lie groups, and more"

VLADIMIR G. PESTOV

Correction to: "Asymptotic radial symmetry for solutions of $\Delta u+e^{u}=0$ in a punctured disc"

KAI Seng (KAISing) CHOU (Tso) and Tom YAU-Heng WAN 